\title{
The site-specific integration of genetic elements may modulate thermostable protease production, a virulence factor in Dichelobacter nodosus, the causative agent of ovine footrot
}

\author{
Gabrielle Whittle, Garry A. Bloomfield, $\uparrow$ Margaret E. Katz \\ and Brian F. Cheetham
}

\begin{abstract}
Author for correspondence: Brian Cheetham. Tel: +6126773 3394. Fax: +61267733267. e-mail: bcheetha@metz.une.edu.au
\end{abstract}

Molecular and Cellular Biology, School of Biological Sciences, University of New England, Armidale, NSW 2351, Australia
The Gram-negative anaerobe Dichelobacter nodosus is the causative agent of footrot in sheep. The authors have previously characterized two genetic elements, the int $A$ (vap) and intB elements, which integrate into the genome of $D$. nodosus. In the virulent strain A198 there are two copies of the intA element. One copy is integrated into the 3' end of the tRNA-ser ${ }_{\mathrm{Gcu}}$ gene, close to the aspartokinase (askA) gene, and the second copy is integrated into the $3^{\prime}$ end of the $t R N A-$ ser $_{\mathrm{GGA}}$ gene, next to the polynucleotide phosphorylase (pnpA) gene. In this study, a new genetic element was identified in the benign strain C305, the intC element, integrated into the $3^{\prime}$ end of the tRNA-ser $_{\mathrm{GCU}}$ gene, next to askA. The intC element was found in most $D$. nodosus strains, both benign and virulent, which were examined, and was integrated into tRNA-ser $_{\mathrm{Gcu}}$ in most strains. Between the askA and tRNA-ser $_{\mathrm{GCu}}$ genes, a gene (designated g/pA), was identified whose predicted protein product has very high amino acid identity with RsmA from the plant pathogen Erwinia carotovora. RsmA acts as a global repressor of pathogenicity in $E$. carotovora, by repressing the production of extracellular enzymes. In virulent strains of $D$. nodosus the int $A$ element was found to be integrated next to pnpA, and either the int $A$ or intC element was integrated next to glpA. By contrast, all but one of the benign strains had int $B$ at one or both of these two positions, and the one exception had neither intA, intB nor intC at one position. The loss of the intC element from the virulent strain 1311 resulted in loss of thermostable protease activity, a virulence factor in $D$. nodosus. A model for virulence is proposed whereby integration of the int $A$ and intC genetic elements modulates virulence by altering the expression of $g / p A$, pnpA, tRNA-ser ${ }_{\mathrm{GCu}}$ and $t R N A$ ser $_{\text {GGA }}$.

Keywords: footrot, virulence, pathogenicity, vap, pathogenicity island

\section{INTRODUCTION}

The Gram-negative anaerobic bacterium Dichelobacter nodosus is the principal causative agent of ovine footrot

† Present address: Roche Diagnostics Australia Pty Ltd, 31 Victoria Avenue, Castle Hill, NSW 2154, Australia.

Abbreviation: HSL, N-(3-oxohexanoyl)-L-homoserine lactone.

The GenBank accession number for the intC element in strain C305 beginning at the left-most EcoRI site (Fig. 1) is Y15939.
(Beveridge, 1941). D. nodosus strains are classified as virulent, intermediate or benign depending on the severity of the disease which they cause in sheep. The vap regions of the $D$. nodosus genome arose by the integration of a genetic element, the int $A$ (vap) element, into a $t R N A$ gene. This element is found in almost all virulent strains, but is absent from the majority of benign strains (Katz et al., 1991; Rood et al., 1996), suggesting that the vap sequences may have a role in virulence. 
Multiple copies of the int $A$ element, termed vap regions 1,2 and 3, are present in the virulent D. nodosus strain A198 (Katz et al., 1994). Regions 1 and 3 form part of a contiguous $11.9 \mathrm{~kb}$ virulence-associated region (Fig. 1; Cheetham et al., 1995). Region 1 is integrated into a $t R N A$-ser ${ }_{\mathrm{GCU}}$ gene close to the aspartokinase (askA) gene, while region 2 is integrated into a different $t R N A$ ser gene, $t R N A-s_{\text {Ger }}{ }_{\mathrm{GA}}$, next to the polynucleotide phosporylase ( $p n p A)$ gene (Bloomfield et al., 1997).

The genes which have been identified in the vap regions of the virulent D. nodosus strain A198 are designated int $A$, vap (virulence-associated protein) $A-H$, and toxA (Fig. 1, Katz et al., 1992; Cheetham et al., 1995; Bloomfield et al., 1997). The int A gene product is an integrase belonging to the lambda family of sitespecific recombinases (Cheetham et al., 1995). VapA and ToxA show amino acid similarity to the HigA and $\mathrm{HigB}$ proteins from the killer plasmid Rts1 (Tian et al., 1996), which encode a toxin and antidote molecule, respectively, and act to prevent loss of the plasmid Rts1 in Escherichia coli. VapA and ToxA may have a similar function in D. nodosus (Bloomfield et al., 1997). The $v a p B$ and $v a p C$ genes are similar to $v a g C$ and $v a g D$ from the virulence plasmid of Salmonella dublin. The products of these genes are believed to coordinate replication of the virulence plasmid with cell division (Pullinger \& Lax, 1992). VapD and VapE have amino acid similarity to the products of plasmid-borne genes of unknown function from a variety of bacterial species (Katz et al., 1992; Cheetham et al., 1995). The remaining genes, vapF, vapG and $v a p H$, have no similarity to previously identified genes.

A native plasmid which contains vap region 1 and an insertion sequence (IS1253) has been isolated from strain AC3577 of D. nodosus, and may be the progenitor of the chromosomal vap sequences found in other strains (Billington et al., 1996). IS1253 is not found in the vap regions of strain A198, but there is a copy near the outermembrane protein (omp) gene cluster, which is responsible for production of the major outer-membrane protein of D. nodosus (Moses et al., 1995).

A second genetic element, the int $B$ element, which may be part of a prophage or a conjugative transposon, is integrated next to vap region 3 in strain A198 (Bloomfield et al., 1997). The end of this element contains an integrase gene, int $B$, a regulatory gene, $\operatorname{reg} A$ (Fig. 1), and three genes of unknown function, gep $A$, gep $B$ and gepC.

In this paper, we describe a new genetic element, the intC element, in D. nodosus. In strain C305, the intC element is integrated into $t R N A$-ser ${ }_{\mathrm{GCU}}$, next to askA. We show that loss of this element from one virulent strain resulted in a concomitant loss of thermostable protease activity, a virulence factor in D. nodosus (Depiazzi et al., 1990). Mechanisms by which the loss of the int $\mathrm{C}$ element might result in the loss of thermostable protease activity are discussed.

\section{METHODS}

General methods. Methods for the growth of D. nodosus, preparation of genomic DNA, cloning and analysis of DNA, Southern blotting, DNA sequencing and DNA sequence analysis have been reported elsewhere (Katz et al., 1994; Bloomfield et al., 1997).

Dichelobacter nodosus strains. Strains A198, AC390, B1006, C305, D1172, G1220, H1204, H1215, 1469, 1493 and 3138 were obtained from Professor J. I. Rood, Monash University, Clayton, Victoria 3168, Australia, and strains 819, 1169, 1311 and 2483 from Dr J. Searson, NSW Agriculture, Private Mail Bag, Yanco, NSW 2703, Australia.

Preparation and screening of a library of genomic DNA from $D$. nodosus strain C305. Genomic DNA from D. nodosus strain C305 was partially digested with the restriction endonuclease Sau3AI and fragments were ligated into the XhoI half-site arms of bacteriophage lambdaGEM-12 (Promega), packaged according to the directions of the manufacturer (Promega), and used to infect the lambda-sensitive E. coli strain LE392 (Sambrook et al., 1989). The library was screened by plaque-lift hybridization, on nitrocellulose membranes, using digoxigen-labelled probes and a chemiluminescent detection kit (Boehringer Mannheim), according to the manufacturer's instructions. DNA was prepared from the lambda clones by the method of Ausubel et al. (1989).

Oligonucleotide primers. Primer sequences were: primer A (askA) TAGAAGCCACGCATGTTACG; primer B (intC) AGCGGTTGTTCCCACATAAA; and primer C (intB) ATAACGCTGGCTTTAGGTGA. PCR conditions have been described previously (Bloomfield et al., 1997).

Assay for protease thermostability. The supernatant from liquid cultures of $D$. nodosus was assayed for proteolytic activity by measuring the release of a soluble blue dye from hide powder azure (Green, 1985). Thermostability was determined in triplicate by measuring the activity before and after heating the supernatant at $60^{\circ} \mathrm{C}$ for $10 \mathrm{~min}$.

\section{RESULTS AND DISCUSSION}

\section{Isolation from strain C305 of sequences flanking vap regions 1 and 3 in strain A198}

The benign strain C305 does not contain the genes vapA-D or toxA, suggesting that the int $A$ element is not present in this strain. However, a HindIII-NruI fragment located close to the left-hand end of vap region 3 (Fig. 1) in strain A198 has been shown previously to hybridize to genomic DNA from strain C305 (Cheetham et al., 1995), and repeated sequences found immediately to the left of $v a p D$ in strain A198 are also present in the benign strain C305 (Katz et al., 1994).

To investigate the hypothesis that the vap regions of strain A198 resulted from integrations of the int A element into the $t R N A$-ser ${ }_{\mathrm{GCU}}$ gene located between the ask $A$ and int $B$ genes (Fig. 1), we prepared a library of genomic DNA from strain C305, and screened it with probes 1, 3 and 4A (Fig. 1). Probe 1 contained part of the ask $A$ gene, flanking the left-hand end of vap region 1 in virulent strain A198; probe 4A contained part of the int $B$ gene flanking the right-hand end of vap region 3 in strain A198; and probe 3 contained the HindIII-NruI fragment from the left-hand end of vap region 3 in strain 


\section{Virulent Strain A198}
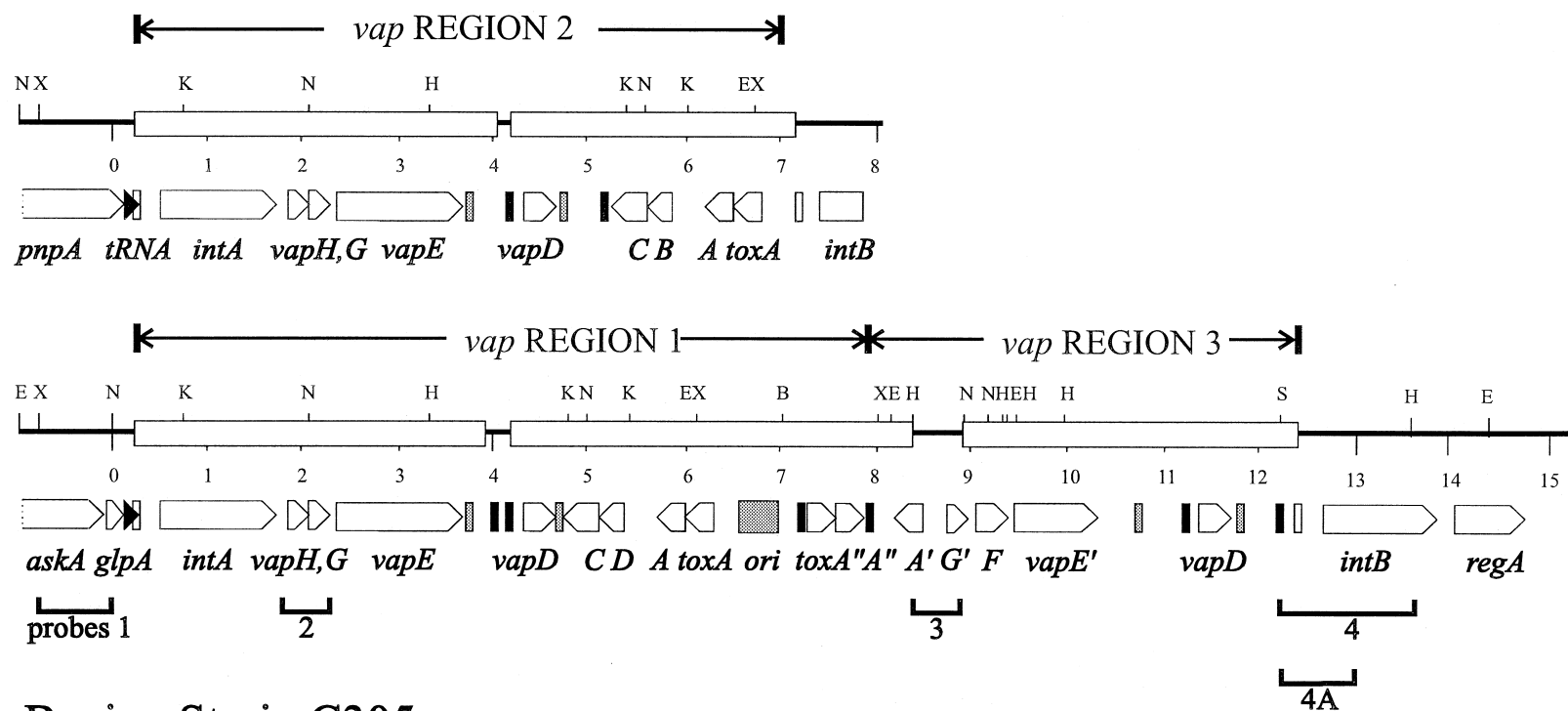

\section{Benign Strain C305}

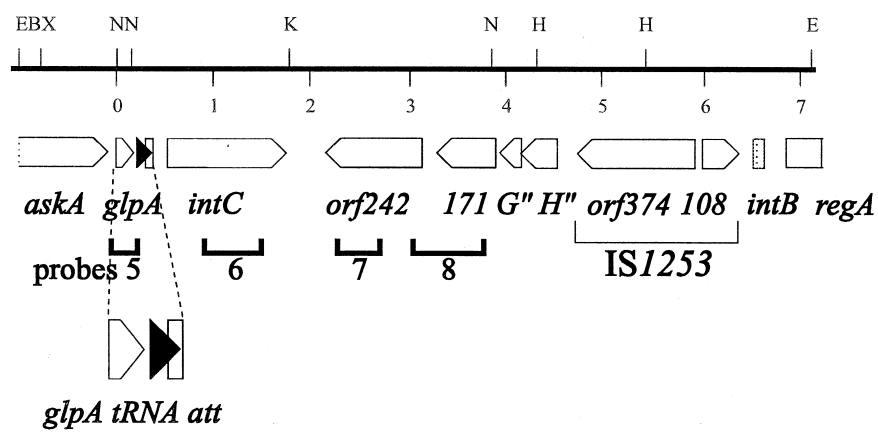

Fig. 1. vap regions 1,2 and 3 of $D$. nodosus strain $A 198$ compared with the intC element in $D$. nodosus strain $C 305$. The numbers show the distance in kb from the left-most Nrul site in strains A198 or C305. Restriction sites shown are BamHI (B), EcoRI (E), HindIII (H), Kpnl (K), Nrul (N), Sacl (S) and Xhol (X). The major potential genes are indicated by open arrows. The tRNA-ser genes are shown by black triangles. Repeated sequences (Cheetham et al., 1995) are indicated as follows: $19 \mathrm{bp}$ att sites (small open boxes), $103 \mathrm{bp}$ repeats, or partial copies thereof (small shaded boxes), $102 \mathrm{bp}$ repeats, or partial copies (small black boxes) and the putative origin of replication (large shaded box). The large open boxes show regions which are not found in strain C305.

A198. A single lambda clone to which probes 1 and 3 hybridized strongly, and probe $4 \mathrm{~A}$ hybridized weakly, was isolated (Fig. 1). We predicted that the askA and int $B$ genes would be close together in this clone. However, they were found to be separated by a $6.6 \mathrm{~kb}$ DNA segment. The DNA sequence of an 8035 bp region from this lambda clone was determined, and several ORFs were identified (Fig. 1).

\section{A new genetic element is integrated into tRNA-ser in strain C305}

At the left-hand end of the lambda clone from strain C305 (Fig. 1), we identified a partial ORF, designated askA, the DNA sequence of which is almost identical to that of the partially characterized ORF located upstream of the $t R N A-s e r_{\mathrm{GCU}}$ gene at the left-hand end of vap region 1 in D. nodosus strain A198 (Bloomfield et al., 1997). Downstream from the askA gene, we identified a 92 bp $t R N A$-ser gene which had $97.8 \%$ nucleotide identity to $t R N A-$ ser $_{\mathrm{GCU}}$ from the left-hand end of vap region 1 of D. nodosus strain A198 (Cheetham et al., 1995).

The predicted protein product from an ORF, designated int $C$, located $200 \mathrm{bp}$ downstream from $t R N A-\operatorname{ser}_{\mathrm{GCU}}$, was found to have $54 \cdot 2 \%$ amino acid identity with Int A from vap region 1 of D. nodosus strain A198 (Fig. 2a). The conserved residues included $\mathrm{Arg}^{251}, \mathrm{His}^{336}$, $\mathrm{Arg}^{339}$ and $\mathrm{Tyr}^{374}$, which are conserved in integrases from this family (Argos et al., 1986; Abremski \& Hoess, 1992). The highest amino acid identity to other integrases from the databases was $40.4 \%$ with the integrase from retronphage $\Phi \mathrm{R} 73$ (Sun et al., 1991). 
(a)

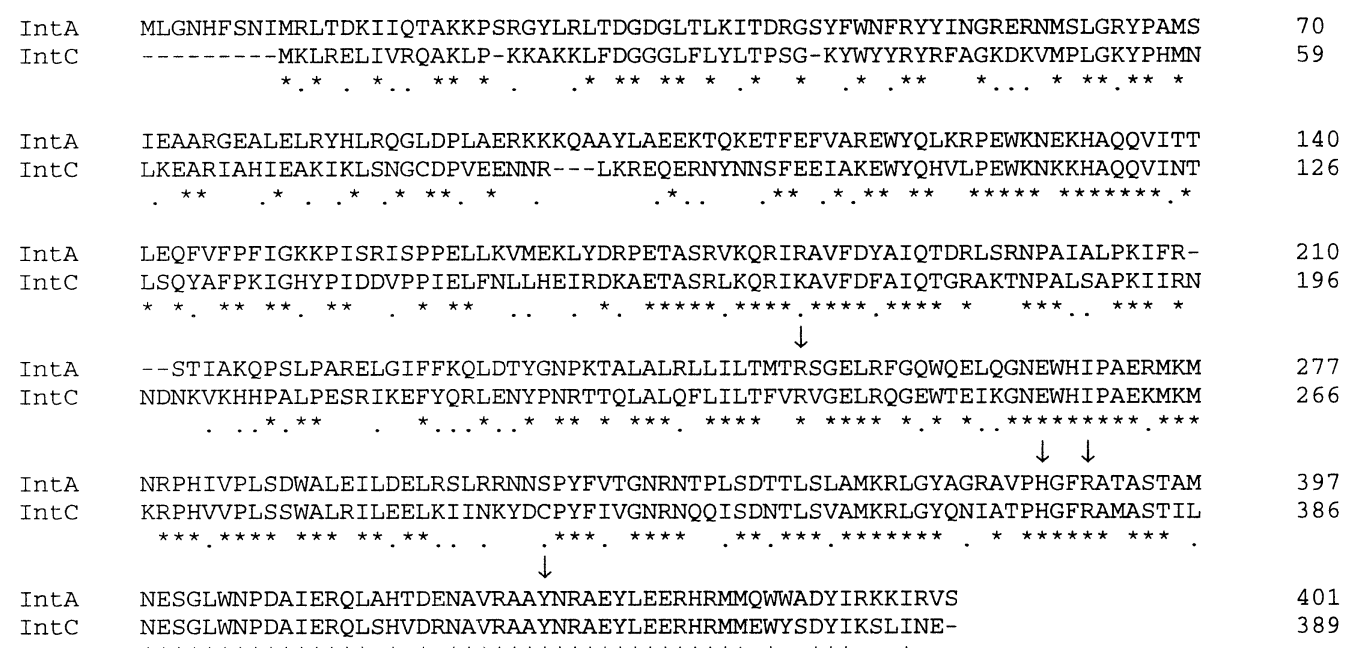

(b)

RSMA MLILTRRVGETLIIGDEVTVTVLGVKGNQVRIGVNAPKEVSVHREEIYQRIQAEKSQPTSY

GIPA MLILTRRVGETIIIDDQIKVTVLAVKGNQVRLGVQAPDEIAIHREEIYHRLMNGVGDDAEMEKK

Fig. 2. Comparison of the amino acid sequences of (a) IntA with IntC, and (b) Er. carotovora RsmA with D. nodosus GIpA. The amino acid residues which are identical $(*)$, amino acids with conservative substitutions $($.$) and amino acids conserved$ among many bacteriophage integrases $(\downarrow)$ are marked.

Two novel ORFs, designated orf242 and orf171, were located downstream from intC in strain C305 (Fig. 1). These two ORFs had no similarity to known genes from the vap regions of $D$. nodosus, nor to any sequences in the databases. These were followed by two small ORFs, designated $v a p G^{\prime \prime}$ and $v a p H^{\prime \prime}$, which showed high similarity to vapG and $v a p H$ from vap region 1 of strain A198. The deduced amino acid sequence of vap $G^{\prime \prime}$ had $55 \cdot 2 \%$ identity with VapG and $\mathrm{VapH}^{\prime \prime}$ had $56 \cdot 2 \%$ amino acid identity with $\mathrm{VapH}$.

\section{IS1253 is next to vapH"}

The insertion sequence IS1253 from D. nodosus strain AC3577 is a $1689 \mathrm{bp}$ element consisting of two ORFs, orf 375 and orf117, with similarity to transposase genes from a number of unusual IS elements (Billington et al., 1996). In strain C305, we found a copy of this element next to vapH" (Fig. 1). The IS1253 found in strain C305 had DNA identity of $98.3 \%$ over $1649 \mathrm{bp}$ with the IS1253 from strain AC3577, and consisted of two similar ORFs, orf374 and orf108. The copy of IS1253 in strain C305 appeared to be non-functional, as the coding region from orf374 had been disrupted by two frameshift mutations, one of which was the deletion of a GTAA repeat. A related insertion sequence is found adjacent to virulence-associated genes in several pathogenic bacteria, including Salmonella typhimurium (Gulig et al., 1992), Helicobacter pylori (Censini et al., 1996), Vibrio cholerae (Bik et al., 1996), Clostridium perfringens (Katayama et al., 1995) and Yersinia pestis (Hu et al., 1998).

\section{The int $B$ and regA genes are disrupted in strain $\mathrm{C} 305$}

The int $B$ and $\operatorname{reg} A$ genes from strain A198 are located immediately to the right of vap region 3 , and form part of the int $B$ genetic element, separate from the int $A$ element (Bloomfield et al., 1997). In strain C305, an internal fragment of the int $B$ coding region was located immediately following IS1253 (Fig. 3a) - a 100 bp DNA segment with $98 \%$ identity to nucleotides $410-510$ of the $1203 \mathrm{bp}$ coding region of the int $B$ gene next to region 3 of strain A198 (Fig. 3b). At this position in the intB gene seven nucleotides from the $19 \mathrm{bp}$ attachment (att) site found at the ends of the vap regions were absolutely conserved (Fig. 3b). Thus, this deletion may have been catalysed by int $C$.

Following the remnant of intB in strain C305, there was a $344 \mathrm{bp}$ segment which had no similarity to other known sequences from $D$. nodosus, or to other sequences from the databases. After this, the DNA sequence from strain C305 was almost identical to the DNA sequence from the beginning of the $\operatorname{reg} A$ gene of strain A198 (Fig. 3c). However, the promoter, ShineDalgarno sequence, and start codon of the regA gene were absent from strain C305. The next 322 bp of the sequence were almost identical, except that there was a 1 bp insertion in the sequence from strain C305, resulting 
(a)

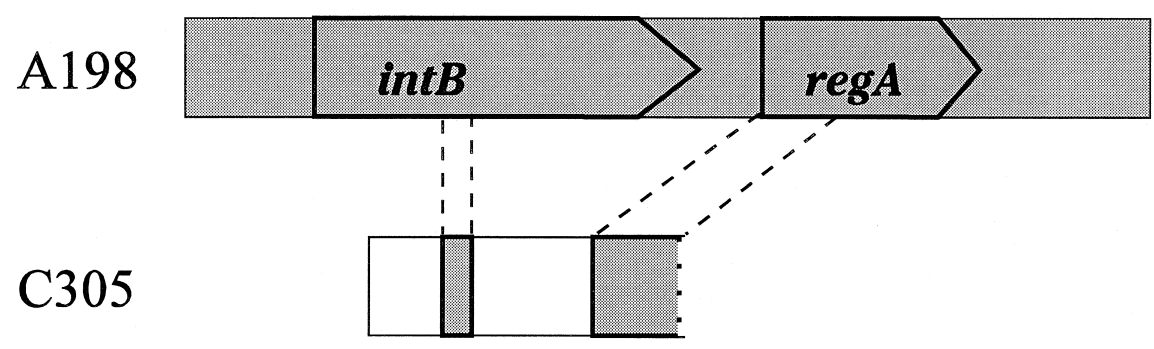

(b)

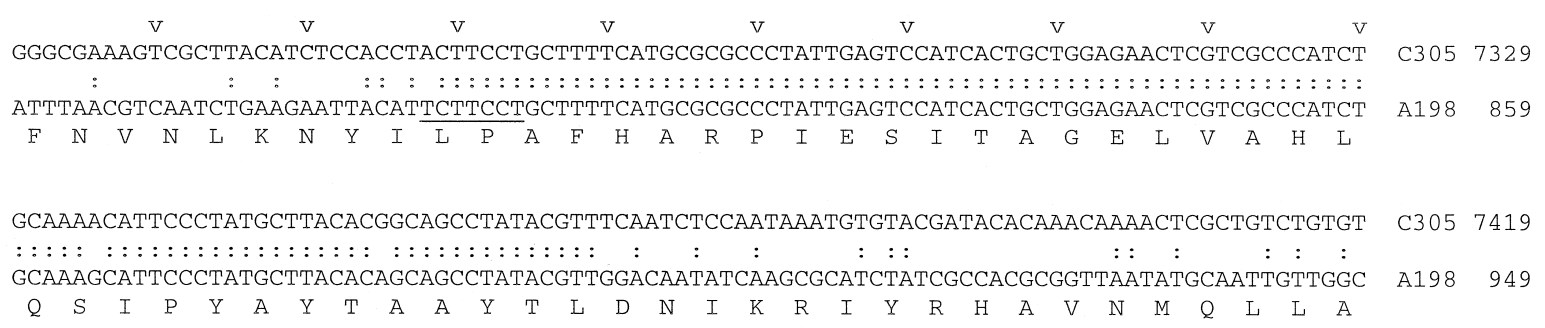

(c)

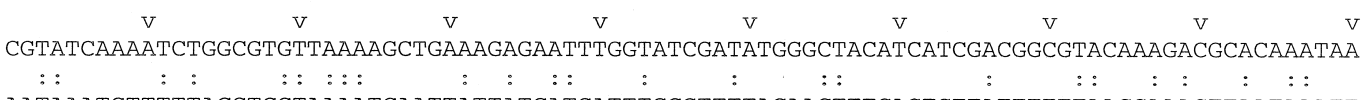

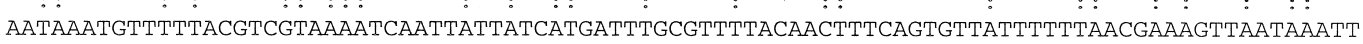

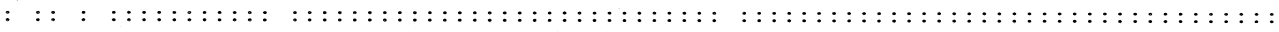
GGTGTTGAAATGAGTCTAGCTAGCAACGTAAAAATATTGCGCGAATTGAATAATCTGTCACAAGACCAACTCGCTGAAAAAATTGGTAAA $\begin{array}{lllllllllllllllllllllllllllllllll}M & S & L & A & S & N & V & K & I & L & R & E & L & N & N & L & S & Q & D & Q & L & A & E & K & I & G & K\end{array}$

TCACAAGCGGCGATTCAAAAAATTGAAGCGGGTTTGACGCTGCGACCGCGTTTTTTGCAAGATTTGGCTAACGCGTTAGGCGTTTCAAGT

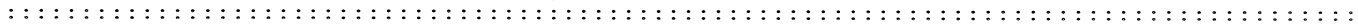
TCACAAGCGGCGATTCAAAAAATTGAAGCGGGTTTGACGCTGCGACCGCGTTTTTTGCAAGATTTGGCTAACGCGTTAGGCGTTTCAAGT

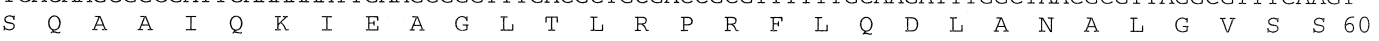

ATTGATTTAGAATATAAAGATTTCGAAAAGAATTGAAGAAACAAGCTATTGAAAGCGATATCGGCACAATGGGCAAATTCCGGCTTTGG

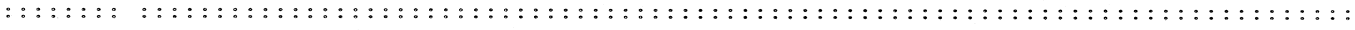
ATTGATTTGGAATATAAAGATTTCGAAAAAGAATTGAAGAAACAAGCTATTGAAAGCGATATCGGCACAATGGGCAAATTCCGGCTTTGG

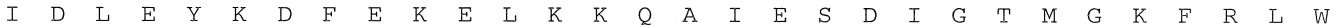

AGCAGTAACGACCCGTTGCCTGAAGACGAATATGCTTATTTGCCGTTTTCAAAGATGTTGAATTC $:::::::::::::::::::::::::::::::::::::::::::::::::::::::::::::::::::::$ : AGCAGTAACGACCCGTTGCCTGAAGACGAATATGCTTATTTGCCGTTTTTCAAAGATGTTGAATTCCAAGGCGGAACAGGTTGCTGCGAA

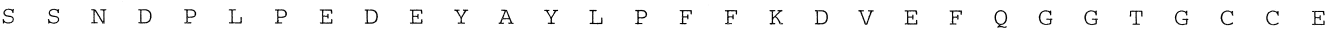

Fig. 3. Comparison of the DNA sequences of strain $C 305$ and strain $A 198$ containing the int $B$ gene and the regA gene. (a) Diagrammatic representation of the int $B$ and regA genes from strains $A 198$ and C305. (b) Comparison of sequences containing the int $B$ gene. (c) Comparison of sequences containing the regA gene. Nucleotides which are identical in both sequences are joined by colons. Every tenth nucleotide is indicated by $v$. The seven nucleotides underlined in (b) are identical to seven nucleotides from the att site.

in a frameshift mutation. Hence, the $\operatorname{reg} A$ gene, like the int $B$ gene, did not appear to be intact in strain C305.

\section{Detection of the intC element in other strains of $D$. nodosus}

To determine whether the genes intC, orf242, orf171, $v a p G^{\prime \prime}$ and $v a p H^{\prime \prime}$ were part of an integrated genetic element, 15 strains of $D$. nodosus were analysed in Southern blot experiments using probes $6,7,8$ and 2 (Fig. 1), which detect intC, orf242, orf171 and $v a p G^{\prime \prime} H^{\prime \prime}$, respectively. The strains included five virulent strains, one intermediate strain, seven benign strains lacking the int $A$ element, and two benign strains which contain the intA element. The results (data not shown) showed that, in ten of these strains, these five genes were 
Table 1. Genetic elements integrated next to ask $A$ and $p n p A$ in 16 strains of $D$. nodosus

\begin{tabular}{|c|c|c|c|}
\hline Strain & Virulence classification & Located next to ask $A$ & $\begin{array}{l}\text { Located next to } \\
\qquad p n p A\end{array}$ \\
\hline A198 & virulent & $\operatorname{int} A$ & int $A$ \\
\hline B1006 & virulent & int $\mathrm{C}$ & int $A$ \\
\hline D1172 & virulent & int $\mathrm{C}$ & int $A$ \\
\hline G1220 & virulent & int $C$ & int $A$ \\
\hline 1311 & virulent & int $\mathrm{C}$ & int $A$ \\
\hline H1215 & virulent & int $B$ & int $A /$ unknown* \\
\hline AC390 & benign & int $B$ & int $A$ \\
\hline C305 & benign & int $C$ & int $B$ \\
\hline H1204 & benign & int $B$ & int $A$ \\
\hline 819 & benign & int $\mathrm{C}$ & int $B$ \\
\hline 1169 & benign & int $B$ & int $B$ \\
\hline $1311 \mathrm{~A}$ & benign & int $B$ & int $A$ \\
\hline 1469 & benign & int $\mathrm{C}$ & int $B$ \\
\hline 1493 & benign & int $\mathrm{C}$ & int $B$ \\
\hline 2483 & benign & int $\mathrm{C}$ & int $B$ \\
\hline 3138 & benign & int $\mathrm{C}$ & not int $A$, int $B$, int $C$ \\
\hline
\end{tabular}

$*$ Two copies of $p n p A$ in this strain.

\section{A B C D}

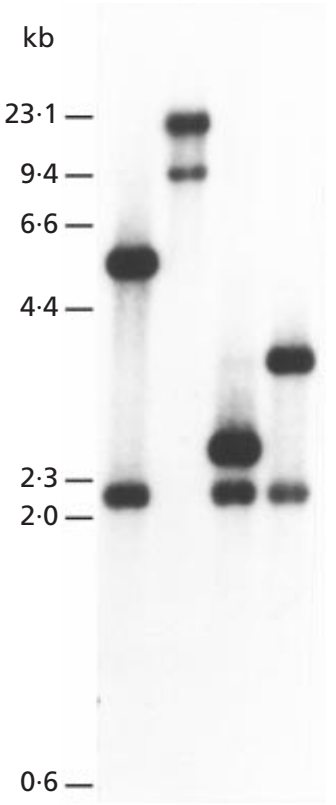

Fig. 4. Southern blot analysis of the int $B$ genes of four strains of $D$. nodosus. Genomic DNA from strains A198 (lane A), C305 (B), 1311 (C) and 1311A (D) was digested with EcoRI and hybridized with a probe specific for intB (probe 4, Fig. 1).

present and found on the same-sized restriction fragment. The virulent strain A198 and the benign strain AC390 contained none of these genes. Since these genes included an integrase gene, were all located together in most strains, and were all absent from both strains A198 and AC390, these data suggest that they are part of an integrated genetic element, which we have designated the int $\mathrm{C}$ element. The virulent strain $\mathrm{H} 1215$ contained two copies of int $C$ and one copy of orf242, but lacked orf171, vap $G^{\prime \prime}$ and vapH", while benign strains H1204 and 1169 contained only int $C$, suggesting that integration of this new genetic element may have been followed by deletion of some of the genes.

\section{Site of integration of the intC element}

The int $\mathrm{C}$ element in strain C305 was integrated into the $t R N A-$ ser $_{\mathrm{GCU}}$ gene downstream from askA (Fig. 1). In the virulent strain A198, vap region 1 is integrated into this $t R N A$-ser gene, while vap region 2 is integrated into $t R N A-$ ser $_{\mathrm{GGA}}$, downstream from the $p n p A$ gene (Bloomfield et al., 1997). Probes specific for askA and pnpA were used in Southern blot experiments to determine the site of integration of the int $C$ element in 13 strains of $D$. nodosus known to contain intC (Table 1: all strains except A198, AC390 and 1311A). In 10 of these strains, the int $C$ element was located next to the ask $A$ gene. None of the strains tested had the int $C$ element located next to pnpA. In strains H1215, H1204 and 1169 , the int $C$ element was not located next to ask $A$, and its site of integration is unknown. These three strains did not have all of the genes so far recognized as being part of the int $C$ element, perhaps suggesting that the int $\mathrm{C}$ element was unstable in the alternative position.

\section{Loss of the intC element from virulent $D$. nodosus strain 1311}

The virulent strain 1311 carries a small native plasmid, pDN1 (G. Whittle, M. E. Katz \& B. F. Cheetham, unpublished), which has not been found in any other strain of D. nodosus. During routine laboratory growth, 
pDN1 was lost spontaneously from strain 1311, generating a strain designated 1311A. Southern blot analysis (data not shown) showed that the int $C$ element was not present in strain 1311A, since genomic DNA from this strain did not hybridize to DNA fragments from int $C$, orf 242 or orf171. vap $G^{\prime \prime}$ and vapH" were also lost, together with one copy of IS1253, Thus, loss of pDN1 was accompanied by loss of the int $C$ element. The concomitant loss of intC, orf242, orf171, vap $G^{\prime \prime}, v a p H^{\prime \prime}$ and a copy of IS1253 provided further evidence that these genes were part of an integrated genetic element.

\section{int $B$ is next to ask $A$ in strain 1311A}

If in strain 1311 the int $B$ element were located next to the int $C$ element, as in strain $\mathrm{C} 305$, then excision of the int $C$ element in strain 1311 would place int $B$ next to ask $A$. Southern blot analysis of strains 1311 and 1311A using an int $B$ probe showed that this was indeed the case (Fig. 4). Both strains contained two copies of intB, one of which is located in a different position in the two strains. In strain 1311, the int $B$ probe hybridized to a $2.5 \mathrm{~kb}$ EcoRI fragment, while in strain $1311 \mathrm{~A}$ a $3 \cdot 3 \mathrm{~kb}$ EcoRI fragment was detected (Fig. 4, lanes C and D). In strain $1311 \mathrm{~A}$, the askA probe also hybridized to a $3.3 \mathrm{~kb} E c o$ RI fragment. These results indicated that in strain 1311, int $C$ was located next to ask $A$ and $\operatorname{int} B$ was located at the right-hand end of the int $C$ element. The loss of the int $C$ element from 1311 has resulted in the relocation of the int $B$ gene such that it was adjacent to ask $A$ in strain 1311A.

In both strains 1311 and $1311 \mathrm{~A}$ the int $A$ element was integrated into $t R N A-s^{-r_{G G A}}$, next to $p n p A$, and a truncated copy of int $B$ was integrated into the att $R$ at the right-hand end of the int $A$ element. The int $B$ probe hybridized to a $2 \cdot 2 \mathrm{~kb} E c o$ RI fragment in both strains, indicating that the position of this copy of intB was unchanged by the excision of the int $C$ element.

To confirm that int $C$ was adjacent to ask $A$ in $D$. nodosus strain 1311, and that int $B$ was adjacent to ask $A$ in strain 1311A, PCR products were amplified using primer sets specific for askA-int C (primers $\mathrm{A}$ and $\mathrm{B}$ ), and ask $A-$ int $B$ (primers $A$ and $C$ ). As expected, a $1.1 \mathrm{~kb}$ askA-int $B$ product was detected in strain $1311 \mathrm{~A}$ whilst no askA-intC product was observed. In strain 1311, a $2.6 \mathrm{~kb}$ askA-int $\mathrm{C}$ product was observed, as expected.

\section{Continuing loss of the intC element from strain 1311}

Contrary to expectations, a $1 \cdot 1 \mathrm{~kb}$ ask $A-i n t B$ product was also obtained in strain 1311, suggesting that int $B$ was also located next to ask $A$ in this strain. However, when serial dilutions of 1311 template and 1311A template were used, the amount of the askA-intB product was much lower in 1311 than in 1311A (data not shown). This suggested that a subpopulation of cells from $D$. nodosus strain 1311 was undergoing loss of the int $C$ element frequently enough to be detected. This was supported by Southern blot analysis (Fig. 4), where a faint band was detected in genomic DNA of strain 1311 by the int $B$ probe at $3.3 \mathrm{~kb}$. The loss of the int $C$ element may be favoured under laboratory conditions.

\section{Excision of the intC element does not disrupt tRNA- ser $_{\mathrm{GCU}}$}

The askA-intB PCR product from strain $1311 \mathrm{~A}$ was subcloned, and the DNA sequence determined and aligned to the left end and right end of vap region $1 / 3$ in D. nodosus virulent strain A198. Analysis of this junction region showed that the left-hand side of the att site was identical to attL, as would be expected if the int $\mathrm{C}$ element were excised by homologous recombination or an integrase-catalysed reaction. Analysis of the DNA sequence also showed that the $t R N A$ gene was not disrupted by the loss of the int $C$ element. Disruption of the leuX $t R N A$ gene by the deletion of a pathogenicity island has been shown to have a major effect on virulence in uropathogenic E. coli (Ritter et al., 1995).

\section{The intC element in strain C305 may be truncated}

Southern blot analysis (summarized in Fig. 5) showed that the distance between ask $A$ and int $B$ in strain 1311 was greater than $14 \mathrm{~kb}$, and that all of the sequences between $t R N A$-ser ${ }_{\mathrm{GCU}}$ and intB were lost together from strain 1311 to form strain 1311A. By contrast, in strain $\mathrm{C} 305$, the distance between ask $A$ and int $B$ was $6.6 \mathrm{~kb}$ (Fig. 1). These results suggested that in strain C305 part of the int $C$ element had been deleted, perhaps due to the insertion of IS1253, which was located at the right-hand end of the intC element in strain C305.

\section{Loss of the intC element from strain 1311 is associated with loss of a virulence characteristic}

It has been established that the extracellular proteases of virulent strains of $D$. nodosus are more thermostable than proteases of benign strains (Depiazzi et al., 1990). The thermostability of the secreted proteases of virulent strain 1311 and its daughter strain 1311A was compared with that of the virulent strain A198 and the benign strain C305. The percentage loss of activity after heating ( \pm standard deviation) was $29 \cdot 5 \pm 5 \cdot 6$ for virulent strain A198, $74 \cdot 1 \pm 11 \cdot 9$ for benign strain C305, $20 \cdot 1 \pm 11 \cdot 4$ for strain 1311, and $84 \cdot 9 \pm 9 \cdot 0$ for strain 1311A. These results clearly showed that the proteases from strain $1311 \mathrm{~A}$ were much less thermostable than the proteases of strain 1311, and therefore strain 1311A behaved as a benign strain in this assay. These results suggest that the loss of the int $\mathrm{C}$ element was associated with loss of protease thermostability, a trait associated with virulence in D. nodosus. In addition, colonies from strain 1311A were smaller and exhibited reduced exoenzyme activity on solid medium when compared to colonies from strain 1311 (data not shown). Virulent strains of $D$. nodosus have, in general, larger colonies and exhibit greater exoenzyme activity than benign strains (Depiazzi \& Richards, 1985). 


\section{Virulent Strain 1311}

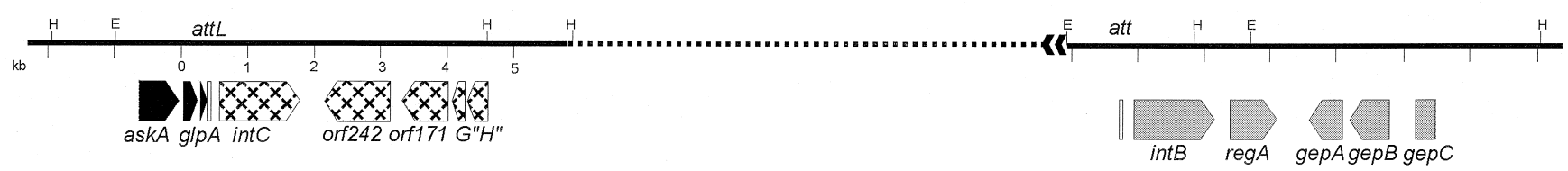

\section{Daughter Strain 1311A}
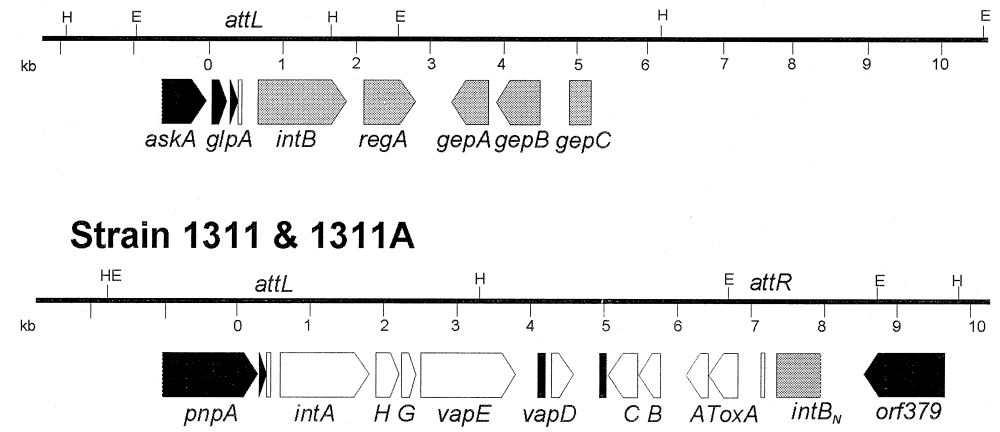

Fig. 5. The intA, intB and intC elements of strains 1311 and $1311 \mathrm{~A}$. Restriction sites shown are EcoRI $(E)$ and $H$ indlII $(H)$. The potential genes are indicated by arrows, which are cross-hatched, shaded, open or black, depending on whether they are part of the int $C$, int $B$ or int $A$ elements, or not part of an integrated genetic element, respectively. The tRNA-ser genes are indicated by black triangles, and the att sites at the left (attL) and right (attR) are indicated by small open rectangles. The DNA sequence of the region shown by a dotted line has not been determined.

\section{Loss of pDN1 may occur without loss of the intC element}

In an effort to determine whether the loss of intC occurred concomitantly with the loss of pDN1, the size difference between colonies of strains 1311 and 1311A was utilized to select colonies of strain 1311 that had potentially lost pDN1 and/or the intC element. Three small colonies were selected, and analysed. All three had lost pDN1 but still contained the intC element, and retained protease thermostability (data not shown). Of the 55 colonies screened, none which had lost the intC element were isolated. These results indicated that the loss of pDN1 may occur without loss of the int C element and that the loss of protease thermostability was not related to the loss of the native plasmid, pDN1, from strain 1311. However, since three colonies of reduced size were found to have lost $\mathrm{pDN} 1$, but not the intC element, the reduced size of $1311 \mathrm{~A}$ colonies may have been due to loss of pDN1.

\section{Sites of integration of the int $A$ and intC elements in virulent and benign strains}

There was no direct correlation between the presence of the int $C$ element and virulence, since the virulent strain A198 does not contain the intC element, while most benign strains do contain it. Southern blot analysis of the integration sites of the int $C$ and int $A$ elements in 16 strains of $D$. nodosus showed that most virulent strains had the int $C$ element next to ask $A$ and the int $A$ element next to pnpA (Table 1). The virulent strain A198 had two copies of int $A$, one next to ask $A$ and one next to $p n p A$. By contrast, most benign strains had int $C$ next to ask $A$, but neither int $C$ nor int $A$ next to $p n p A$. We propose that virulent strains have the int $A$ element next to $p n p A$ and either the int $A$ element or int $C$ element next to askA. Strains which lack the int $A$ element next to $p n p A$, or have neither the int $A$ nor the int $C$ element next to ask $A$, would be benign if our model were correct.

Of the 16 strains studied, the only exception to the above pattern was virulent strain H1215, which has intB next to askA. Strain H1215 was atypical in several ways. Unlike all other strains studied, it contained two copies of $p n p A$, one of which had int $A$ next to it. It is not known what is next to the other copy of $p n p A$. This strain also had two copies of int $C$, and their integration sites are unknown. However, it lacked orf171, vap $G^{\prime \prime}$ and vapH", suggesting that deletions had occurred. Strain H1215 also contains a complete integrated prophage (G. A. Bloomfield, unpublished), but the integration site is also unknown.

\section{A putative global repressor ( $g / p A)$ gene between askA and tRNA-ser ${ }_{\mathrm{GCU}}$}

Between the askA and $t R N A$-ser ${ }_{\mathrm{GCU}}$ genes we identified an ORF, designated $g l p A$ (Fig. 1), encoding a putative product of 64 aa which had $74 \%$ aa identity over the first 50 aa with RsmA (Fig. 2b) from the plant pathogen Erwinia carotovora (Cui et al., 1995). In Er. carotovora, RsmA acts as a global repressor of virulence. The $r s m A$ gene product reduces the transcript level of hslI, a luxI homologue required for $\mathrm{N}$-(3-oxohexanoyl)-L-homoserine lactone (HSL) synthesis (Cui et al., 1995), and 
thereby represses extracellular enzyme production, reduces motility and colony size, and so attenuates virulence (Mukherjee et al., 1996). It is interesting that D. nodosus strain $1311 \mathrm{~A}$ exhibits a similar phenotype to cells expressing rsmA in Er. carotovora, given the similarity between GlpA and RsmA. CsrA from E. coli, which is also very similar to RsmA, modulates glycogen biosynthesis by effects on mRNA stability (Liu et al., 1995). CsrA belongs to a family of proteins containing $\mathrm{KH}$ domains, which are involved in RNA binding. The E. coli pnp gene belongs to the same family (Liu et al., 1995), affects mRNA processing and decay (Alifano et al., 1994), and is one of the two principal enzymes involved in the degradation of bacterial mRNA to nucleotides (Li \& Deutscher, 1994). Thus, in D. nodosus, the int $A$ and the int $C$ elements are integrated next to two genes whose products may be involved in gene regulation by the alteration of mRNA stability. The integration of these genetic elements may affect levels of $g l p A$ and $p n p A$ transcripts, resulting in increased production of thermostable extracellular proteases.

\section{Comparison of DNA sequences at the beginning of the int $A$, int $B$ and int $C$ elements}

Analysis of the DNA sequences at the beginning of the int $A$, int $B$ and int $C$ elements identified several features which may be important in differential gene expression in D. nodosus strains containing different integrated genetic elements. These are discussed below.

Termination of transcription. The DNA sequences starting from the TGA stop codon for $g l p A$ for strains C305, A198 and 1311A were compared (data not shown). In all three strains, the sequence was almost identical up to the $3^{\prime}$ end of the $t R N A$ gene (the attL site). Within this sequence, there was a region of dyad symmmetry (loop 1) very close to the stop codon for $g l p A$, which could act as a transcriptional terminator for $g l p A$. Strains C305, A198 and 1311A had the intC, int A and intB elements, respectively, integrated after the att $L$ site. The first $42 \mathrm{bp}$ of the int $A$ and int $C$ elements were identical, and differed substantially from the first $42 \mathrm{bp}$ of the int $B$ element. Within this 42 bp sequence was a second region of dyad symmetry (loop 2), which could act as an alternative transcriptional terminator for $g l p A$. If this transcriptional terminator were used, the transcript would include the tRNA molecule. This dyad symmetry (loop 2) was not present in the intB element. Thus, termination of transcription of $g l p A$ may differ according to whether int $C$, int $A$ or int $B$ is integrated at this position.

Virulent strains had the int $A$ element integrated next to $p n p A$, while most benign strains had int $B$ at this position. In the virulent strain A198, the stop codon in pnpA was located only one base before the $t R N A$ $\operatorname{ser}_{\mathrm{GGA}}$ gene, and no putative transcriptional terminators were evident before the end of the $t R N A$ gene. Thus, it is likely that $p n p A$ transcripts include the tRNA molecule, and would differ according to the genetic element integrated at this position. The integration of the int $A$ element, but not the int $B$ element, next to $p n p A$ would provide a transcriptional terminator.

Antisense RNA. Within the divergent region downstream of loop 2, sequences weakly resembling the consensus sequence for the E. coli $\sigma^{70}-35$ and -10 promoter sequences (Hawley \& McClure, 1983) were identified in the regions upstream of int $C$ and $i n t A$, but were not present upstream of int $B$. If these promoter sequences are functional, the antisense transcript produced could potentially sequester those messages transcribed on the opposite DNA strand, and left of the int $C$ element and int $A$ element integration sites, including those transcripts for $g l p A, p n p A, t R N A-$ ser $_{\mathrm{GCU}}$ and $t R N A-$ ser $_{\mathrm{GGA}}$ genes.

tRNA processing. Since the int $A$ and int $C$ elements integrate into the $3^{\prime}$ ends of $t R N A-$ ser $_{\mathrm{GGA}}$ and $t R N A$ $\operatorname{ser}_{\mathrm{GCU}}$, they could alter the expression of these genes, thereby affecting the levels of these two serine tRNAs. This could, in turn, affect the translation of genes which use the corresponding serine codons. The tRNA-ser may have a role in virulence, similar to that proposed in uropathogenic E. coli, where the LeuX tRNA molecule is thought to act as a global regulator of several virulence factors (Ritter et al., 1995).

DNA topology. It has been reported previously that the integration of genetic elements can alter gene expression by affecting DNA topology (Dorman, 1995; Ott, 1993; Zagaglia et al., 1991). It is therefore possible that an element of critical size, such as the int $C$ or int $A$ elements, must be integrated adjacent to both $g l p A$ and $p n p A$, in order to provide the appropriate topology for thermostable protease expression or secretion.

\section{A model for the role of the int $A$ and int $C$ elements in the virulence of $D$. nodosus}

Our analysis of 16 strains of $D$. nodosus has shown that the int $A$, int $B$ or int $C$ elements may integrate next to $g l p A$, and either the int $A$ or the int $B$ elements may integrate next to $p n p A$. Our model proposes that virulent strains of $D$. nodosus have the int $A$ element integrated next to the $p n p A$ gene, and the int $C$ or int $A$ element integrated next to the $g l p A$ gene. The presence of these integrated genetic elements may alter the expression of $g l p A, p n p A, t R N A-\operatorname{ser}_{\mathrm{GCU}}, t R N A-$ ser $_{\mathrm{GGA}}$ or askA, and reduce the expression of thermostable proteases through effects on mRNA stability, tRNA availability, or HSL levels.

It has been observed previously that virulent strains may have more than one copy of the int $A$ element, but that strains with increased numbers of copies do not show increased virulence. This is consistent with our model, since the integration of a single copy of the int $A$ element next to the pnpA gene and the int $C$ element next to the $g l p A$ gene is sufficient for virulence. The presence of the int $A$ element in some benign strains is also consistent with this model, since these strains would be benign if they did not have either the int $A$ or the int $C$ element integrated next to $g l p A$, and the int $A$ element integrated 
next to $p n p A$. For example, the benign strain AC390 has two copies of int $A$, one of which is integrated next to $p n p A$, but does not have the intC element, and the second copy of the int $A$ element is not integrated next to glpA (Table 1). Based on these results, a diagnostic test to determine the virulence of strains of $D$. nodosus could be designed, using PCR to determine which genetic elements are integrated next to $g l p A$ and $p n p A$.

There is one report of a small number of virulent strains which lack sequences from the vap region (Rood et al., 1996). These strains may have a copy of the int $C$ element integrated next to both $g l p A$ and $p n p A$. It will be of great interest to analyse such strains to determine whether genetic elements are integrated at these two locations.

\section{Similarities between the int $A$ and int $C$ elements}

The majority of the genes from the int $A$ and int $C$ elements are quite unrelated. However, the two integrases, IntA and IntC, have $54 \cdot 2 \%$ amino acid identity, and $v a p G$ and $v a p H$, from the int $A$ element, and $v a p G^{\prime \prime}$ and $v a p H^{\prime \prime}$, from the intC element, have approximately $55 \%$ amino acid identity, and $81 \%$ nucleotide identity. In addition, two sequences of $42 \mathrm{bp}$ and $61 \mathrm{bp}$ are almost totally conserved in the DNA segment between the att site and the beginning of int $A$ and int $C$. These results suggest that there has been genetic exchange between the int $A$ and int $C$ elements, that the related sequences are required for similar functions and have been maintained by selection, or that the two elements have evolved from a common ancestor.

\section{Phase variation in $D$. nodosus}

In culture we have shown loss of the int $C$ element from a virulent strain of $D$. nodosus, resulting in loss of a virulence factor. Loss of the intC element from $D$. nodosus strains in natural infections may allow switching from the virulent to the benign phenotype, and may have a selective advantage. However, this has not yet been observed in vivo. Loss of the int A element is very unlikely, due to the vapA/toxA maintenance system in this element (Bloomfield et al., 1997). At present, we do not know whether the int $A$, int $B$ or int $C$ elements can be transferred between D. nodosus strains, although a plasmid containing the genes from vap region 1 has been identified in one strain of $D$. nodosus (Billington et al., 1996). It is possible that strains of $D$. nodosus may be able to switch between the benign and virulent states by the loss or gain of these genetic elements. This work has significant implications for footrot eradication programmes which are currently in place in Australia.

\section{ACKNOWLEDGEMENTS}

We thank J. Druitt for her excellent technical assistance, and J. Rood and J. Searson for the provision of D. nodosus strains. This work was supported by a grant from the Australian
Research Council. G.W. and G.B. were recipients of Australian Postgraduate Research Awards.

\section{REFERENCES}

Abremski, K. E. \& Hoess, R. H. (1992). Evidence for a second conserved arginine residue in the integrase family of recombination proteins. Protein Eng 5, 87-91.

Alifano, P., Bruni, C. B. \& Carlomagno, M. S. (1994). Control of mRNA processing and decay in prokaryotes. Genetica 94, 157-172.

Argos, P., Landy, A., Abremski, K. \& 9 other authors. (1986). The integrase family of site-specific recombinases: regional similarities and global diversity. EMBO J 5, 433-440.

Ausubel, F. M., Brent, R., Kingston, R. E., Moore, D. D., Seidman, J. G., Smith, J. A. \& Struhl, K. (1989). Current Protocols in Molecular Biology. New York: Wiley.

Beveridge, W. I. B. (1941). Footrot in sheep: a transmissible disease due to infection with Fusiformis nodosus. CSIRO Bull $140,1-40$.

Bik, E. M., Gouw, R. D. \& Mooi, F. R. (1996). DNA fingerprinting of Vibrio cholerae strains with a novel insertion sequence element: a tool to identify epidemic strains. J Clin Microbiol 34, 1453-1461.

Billington, S. J., Sinistaj, M., Cheetham, B. F., Ayres, A., Moses, E. K., Katz, M. E. \& Rood, J. I. (1996). Identification of a native Dichelobacter nodosus plasmid and implications for the evolution of the vap regions. Gene 172, 111-116.

Bloomfield, G. A., Whittle, G., McDonagh, M. B., Katz, M. E. \& Cheetham, B. F. (1997). Analysis of sequences flanking the vap regions of Dichelobacter nodosus: evidence for multiple integration events, a killer system, and a new genetic element. Microbiology 143, 553-562.

Censini, S., Lange, C., Xiang, Z., Crabtree, J. E., Ghiara, P., Borodovsky, M., Rappuoli, R. \& Covacci, A. (1996). cag, a pathogenicity island of Helicobacter pylori, encodes type Ispecific and disease-associated virulence factors. Proc Natl Acad Sci USA 93, 14648-14653.

Cheetham, B. F., Tattersall, D. B., Bloomfield, G. A., Rood, J. I. \& Katz, M. E. (1995). Identification of a bacteriophage-related integrase gene in a vap region of the Dichelobacter nodosus genome. Gene 162, 53-58.

Cui, Y., Chatterjee, A., Liu, Y., Dumenyo, C. K. \& Chatterjee, A. K. (1995). Identification of a global repressor gene, $r s m A$, of Erwinia carotovora subsp. carotovora that controls extracellular enzymes, $\mathrm{N}$-(3-oxohexanoyl)-L-homoserine lactone, and pathogenicity in soft-rotting Erwinia spp. J Bacteriol 177, 5108-5115.

Depiazzi, L. J. \& Richards, R. B. (1985). Motility in relation to virulence of Bacteroides nodosus. Vet Microbiol 10, 107-116.

Depiazzi, L. J., Henderson, J. \& Penhale, W. J. (1990). Measurement of protease thermostability, twitching motility and colony size of Bacteroides nodosus. Vet Microbiol 22, 353-363.

Dorman, C. J. (1995). DNA topology and the global control of bacterial gene expression: implications for the regulation of virulence gene expression. Microbiology 141, 1271-1280.

Green, R. S. (1985). A method to differentiate between virulent and benign isolates of Bacteroides nodosus based on the thermal stability of their extracellular proteinases. NZ Vet J 33, 11-13.

Gulig, P. A., Caldwell, A. L. \& Chiodo, V. A. (1992). Identification, genetic analysis and DNA sequence of a $7 \cdot 8-\mathrm{kb}$ virulence region of the Salmonella typhimurium virulence plasmid. Mol Microbiol 6, 1395-1411. 
Hawley, D. K. \& McClure, W. R. (1983). Compilation and analysis of Escherichia coli promoter DNA sequences. Nucleic Acids Res 11, 2237-2255.

Hu, P., Elliott, J., McCready, P., Skowronski, E., Garnes, J., Kobayashi, A., Brubaker, R. R. \& Garcia, E. (1998). Structural organization of virulence-associated plasmids of Yersinia pestis. J Bacteriol 180, 5192-5202.

Katayama, S., Dupuy, B., Garnier, T. \& Cole, S. T. (1995). Rapid expansion of the physical and genetic map of the chromosome of Clostridium perfringens CPN50. J Bacteriol 177, 5680-5685.

Katz, M. E., Howarth, P. M., Yong, W. K., Riffkin, G. G., Depiazzi, L. J. \& Rood, J. I. (1991). Identification of three gene regions associated with virulence in Dichelobacter nodosus, the causative agent of ovine footrot. J Gen Microbiol 137, 2117-2124.

Katz, M. E., Strugnell, R. A. \& Rood, J. I. (1992). Molecular characterization of a genomic region associated with virulence in Dichelobacter nodosus. Infect Immun 60, 4586-4592.

Katz, M. E., Wright, C. L., Gartside, T. S., Cheetham, B. F., Doidge, C. V., Moses, E. K. \& Rood, J. I. (1994). Genetic organization of the duplicated vap region of the Dichelobacter nodosus genome. $J$ Bacteriol 176, 2663-2669.

Li, Z. \& Deutscher, M. P. (1994). The role of individual exoribonucleases in processing at the $3^{\prime}$ end of Escherichia coli tRNA precursors. J Biol Chem 269, 6064-6071.

Liu, M. Y., Yang, H. \& Romeo, T. (1995). The product of the pleiotropic Escherichia coli gene csrA modulates glycogen biosynthesis via effects on mRNA stability. J Bacteriol 177, 2663-2672.

Moses, E. K., Good, R. T., Sinistaj, M., Billington, S. J., Langford, C. J. \& Rood, J. I. (1995). A multiple site-specific inversion model for the control of Omp1 phase and antigenic variation in Dichelobacter nodosus. Mol Microbiol 17, 183-196.

Mukherjee, A., Cui, Y., Liu, Y., Dumenyo, C. K. \& Chatterjee, A. K. (1996). Global regulation in Erwinia species by Erwinia carotovora rsmA, a homologue of Escherichia coli csrA: repression of secondary metabolites, pathogenicity and hypersensitive reaction. Microbiology 142, 427-434.

Ott, M. (1993). Dynamics of the bacterial genome: deletions and integrations as mechanisms of bacterial virulence modulation. Zentbl Bakteriol 278, 457-468.

Pullinger, G. D. \& Lax, A. J. (1992). A Salmonella dublin virulence plasmid locus that affects bacterial growth under nutrient-limited conditions. Mol Microbiol 6, 1631-1643.

Ritter, A., Blum, G., Emody, L., Kerenyi, M., Bock, A., Neuhier, B., Rabsch, W., Scheutz, F. \& Hacker, J. (1995). tRNA genes and pathogenicity islands: influence on virulence and metabolic properties of uropathogenic Escherichia coli. Mol Microbiol 17, 109-121.

Rood, J. I., Howarth, P. A., Haring, V. \& 7 other authors. (1996). Comparison of gene probe and conventional methods for the diagnosis of ovine footrot. Vet Microbiol 52, 127-142.

Sambrook, J., Fritsch, E. F. \& Maniatis, T. (1989). Molecular Cloning: a Laboratory Manual, 2nd edn. Cold Spring Harbor, NY: Cold Spring Harbor Laboratory.

Sun, J., Inouye, M. \& Inouye, S. (1991). Association of a retroelement with a P4-like cryptic prophage (retronphage phiR73) integrated into the selenocystyl tRNA gene of E. coli.J Bacteriol 173, 4171-4181.

Tian, Q. B., Ohnishi, M., Tabuchi, A. \& Terawaki, Y. (1996). A new plasmid-encoded killer gene system: cloning, sequencing, and analyzing hig locus of plasmid Rts1. Biochem Biophys Res Commun 220, 280-284.

Zagaglia, C., Casalino, M., Colonna, B., Conti, C., Calconi, A. \& Nicoletti, M. (1991). Virulence plasmids of enteroinvasive Escherichia coli and Shigella flexneri integrate into a specific site on the host chromosome: integration greatly reduces expression of plasmid-carried virulence genes. Infect Immun 59, 792-799.

Received 2 March 1999; revised 10 June 1999; accepted 1 July 1999. 